\title{
Qualitative Methods in Systemic Sclerosis Research
}

Qualitative research methods are important tools that are frequently underused in medical research, particularly in systemic sclerosis (scleroderma, $\mathrm{SSc}$ ) research. In this issue of The Journal, Nakayama, et al report a thematic synthesis of qualitative studies of patients' perspectives and experiences living with $\mathrm{SSc}^{1}$. This editorial serves as a brief introduction to qualitative research, addressing the questions: What is it? How is it used? How is it different from quantitative research? What are key indicators of its rigor?

\section{Quantitative vs Qualitative Research Methods}

Quantitative research is a form of study design that uses statistical methods or other means of quantification to address a research question. It involves deductive approaches and hypothesis testing to approximate the truth and handling of uncertainty ${ }^{2}$. Quantitative methods generally evaluate participants in settings removed from the natural environment (e.g., randomized controlled trials) and attempts are made to control for confounding factors. In contrast, qualitative research is a form of study design that uses systematic and reproducible methods to explore experiences, behaviors, and beliefs about a concept or phenomenon. Qualitative research commonly involves interpretative forms of analysis in which the unit of study is often a participant's experienced reality ${ }^{3}$. Results are a representation of reality rather than an approximation of the truth (see Table 1).

Qualitative methods may be preferable if one wishes to develop a new theory, explore an unknown phenomenon, evaluate the meaning of a concept, or understand a phenomenon. Qualitative research can provide more detailed descriptions and nuanced understanding of a concept, in contrast to a "cause and effect" relationship. A qualitative approach may facilitate a more in-depth understanding of quantitative results, answering "what do these numbers really mean?" For example, unhealthy lifestyle behavior, such as smoking, can be a significant risk factor for disease. However, it is important to understand that the experience of an individual's smoking status is embedded within his/her cultural and social context ${ }^{4}$. Therefore, giving a numeric estimate of the magnitude of association between the unhealthy behavior (e.g., smoking) and the health state can result in an incomplete understanding of the relationship and opportunities for health improvement (e.g., smoking cessation programs). Where quantitative research can address the "how much?" question, qualitative research can address the "why?" and "how?" questions. While quantitative research results are usually represented in the form of numbers, qualitative research results are often represented in the form of quotations of participants describing the experience behind a particular concept of interest. Or investigators might use mixed methods, combining quantitative and qualitative research techniques, into a single study.

Common examples of qualitative data collection methods may include one-on-one interviews, focus groups, video analysis, or participant observation. Interview questions tend to be semistructured in nature, allowing the interviewer to use probing questions to explore in more depth a concept of interest (e.g., Can you tell me more about that?). An interview or focus group discussion guide is commonly the data collection tool that can provide structure to the sequence of the interview while allowing for flexibility. Interviews are usually audio recorded, and later transcribed verbatim. Analysis of qualitative data can include theoretical or descriptive approaches. Descriptive qualitative analysis often involves line-by-line coding of text. Codes are clustered into broader categories that can then comprise an overall theme that collectively describes the concept of interest. Results of qualitative research are often represented in the form of themes, supported by direct quotations from participants to illustrate a concept $t^{5}$. Multimethod studies may combine qualitative data collection strategies such as the combination of focus groups and interviews.

Table 1. Comparison of quantitative and qualitative methods.

\begin{tabular}{ll}
\hline Quantitative & Qualitative \\
\hline Hypothesis-testing (deductive) & Hypothesis-generating (inductive) \\
Cause-effect & Understanding, meaning \\
Random sampling & Purposive sampling \\
Control extraneous variables & Consider extraneous variables \\
Reliability and validity & Dependability and trustworthiness
\end{tabular}

See Living with SSc, page 1363

Personal non-commercial use only. The Journal of Rheumatology Copyright @) 2016. All rights reserved. 


\section{Examples of Qualitative Research in SSc}

The scleroderma research community is using qualitative methods to develop classification criteria for SSc subsets. In an era of antibody profiling, genetics, and molecular classification, the meaning and purpose of classification criteria for SSc subsets is uncertain. Using one-on-one, face-to-face interviews of international SSc experts and a content-analytic approach, investigators found that the concept of SSc subsets is a multidimensional and complex construct ${ }^{6}$. Experts believe that subsets exist and have an effect on the aggressiveness of investigations, and on therapeutic decision making, prognosis, and survival. This information is being used to inform the study design of subsequent quantitative phases of SSc subset classification criteria development.

In the present report by Nakayama, et al ${ }^{1}$ the authors describe a document analysis of qualitative studies among individuals with SSc. The authors performed a systematic review of the literature and conducted a thematic synthesis of 26 articles that used qualitative methods to describe the perspectives and experiences of adults with SSc. Qualitative methods of data collection reported in the literature included face-to-face interviews, focus groups, and an open-ended questionnaire. The authors identified 6 themes that captured the experiences of adults with SSc across the included studies: distressing appearance transformation, palpable physical limitations, social impairment, navigating uncertainty, feeling alone and misunderstood, and gradual acceptance and relative optimism. Under the domain of distressing appearance transformation, the authors reported that "patients felt they looked terrifying, unattractive and undesirable, using terms such as 'freak,' 'dracula,' and 'monster' to describe themselves." Under the thematic domain of gradual acceptance and relative optimism, the authors report: "One patient was touched by her husband who said 'after 30 years of marriage we are the same people inside we just have different wrappers."”

The participant quotations represent the lived experience, and offer a call to action to take a "multidisciplinary approach to patient-centered care that encompasses strategies to promote self-esteem, self-efficacy and open communication ${ }^{2} . "$

The insights gained from thematic synthesis, such as those reported by Nakayama, et al, are critically important if the global SSc community is to develop a patient-centered research agenda. As an example, SPIN (Scleroderma Patient-centered Intervention Network), an international collaboration of patient groups and SSc researchers, has been established to test accessible, low-cost, online interventions to improve quality of life and reduce disability ${ }^{7}$.

\section{Principles of Qualitative Study Design}

The principles of study design such as the sampling frame, sample size, and method of data analysis remain true in qualitative studies.
Sampling frame. Random sampling is frequently encouraged in quantitative research to reduce the risk of potential bias, and to allow for extrapolation of findings to the larger population. Because the goal of qualitative research is to understand an underlying concept, a sample should be chosen to represent a range of perspectives ${ }^{4}$. Preferred sampling strategies include purposive sampling (individuals are chosen based on the needs for the study), convenience sampling (sample chosen based on ease of access), or snowball sampling (respondents nominate other candidates).

Sample size. There are no firm guidelines on an appropriate sample size for a qualitative study. Rather, emphasis is put on saturation, which involves recruitment of sufficient individuals to achieve adequate representation of the concepts, until no new information is gained. However, a sample size justification is necessary, and should include consideration of feasibility, the target population, and typical numbers of participants required to achieve saturation in similar studies ${ }^{8}$.

Analysis. Qualitative content analysis often involves classifying large amounts of text into categories that represent similar meanings ${ }^{9}$. Transcripts of interviews or focus groups are reviewed line by line to identify themes that represent an idea. Codes are assigned to the ideas. Codes are clustered into broader categories or themes. This process is conducted iteratively, while identifying new themes and considering conflicting ideas ${ }^{10}$.

\section{Assessing the Rigor of a Qualitative Study}

Attributes of rigor in qualitative research include trustworthiness, dependability, and transferability (analogous to validity, reliability, and generalizability in a quantitative study). A number of approaches may be undertaken to ensure the trustworthiness of the results. Theoretical saturation can be achieved by continuing to collect data until no new relevant data or themes emerge. Approaches to ensuring analytical rigor may include verifying the accuracy of transcription, coding carried out by 2 or more investigators, and reviewing preliminary results with the participants.

Qualitative research is a broad methodological approach that can be used by investigators to better understand a concept or phenomenon. Application of these methods requires the same considerations and benchmarks for methodologic rigor as quantitative methods. Qualitative methods should be considered part of an investigator's toolkit to improve our understanding of rheumatic diseases, including SSc.

SINDHU R. JOHNSON, MD, $\mathrm{PhD}$,

Toronto Scleroderma Program,

Division of Rheumatology, Department of Medicine, Toronto Western Hospital;

Institute of Health Policy, Management and Evaluation, University of Toronto, Toronto, Ontario, Canada;

Personal non-commercial use only. The Journal of Rheumatology Copyright $\subset$ 2016 . All rights reserved. 
KELLY K. O'BRIEN, PhD,

Department of Physical Therapy, Rehabilitation Sciences Institute,

Institute of Health Policy, Management and Evaluation, University of Toronto,

Toronto, Ontario, Canada.

Address correspondence to Dr. S.R. Johnson, Division of Rheumatology, Ground Floor, East Wing, Toronto Western Hospital, 399 Bathurst St.,

Toronto, Ontario M5T 2S8, Canada. E-mail: Sindhu.Johnson@uhn.on.ca

\section{ACKNOWLEDGMENT}

Dr. Johnson is supported by a Canadian Institutes of Health Research Clinician Scientist Award, and the Oscar and Eleanor Markovitz fund for Scleroderma Research through the Arthritis Research Foundation. Dr. O'Brien is supported by a New Investigator Award from the Canadian Institutes of Health Research.

\section{REFERENCES}

1. Nakayama A, Tunnicliffe DJ, Thakkar V, Singh-Grewal D, O'Neill $\mathrm{S}$, Craig JC, et al. Patients' perspectives and experiences living with systemic sclerosis: a systematic review and thematic synthesis of qualitative studies. J Rheumatol 2016;43:1363-75.

2. Johnson SR, Tomlinson GA, Hawker GA, Granton JT, Feldman BM. Methods to elicit beliefs for Bayesian priors: a systematic review. J Clin Epidemiol 2010;63:355-69.
3. Strauss A, Corbin J. Open coding. Basics of qualitative research. 2nd ed. Thousand Oaks, California: Sage Publications Inc.; 1998.

4. Rowan M, Huston P. Qualitative research articles: information for authors and peer reviewers. CMAJ 1997;157:1442-6.

5. Sandelowski M. Focus on qualitative methods. The use of quotes in qualitative research. Res Nurs Health 1994;17:479-82.

6. Johnson SR, Soowamber M, Fransen J, Khanna D, van den Hoogen $\mathrm{F}$, Baron M, et al. There is a need for new systemic sclerosis subset classification criteria: a content analytic approach. Arthritis Rheum 2015;67:2257-8.

7. Riehm KE, Kwakkenbos L, Carrier ME, Bartlett SJ, Malcarne VL, Mouthon L, et al. Validation of the Self-Efficacy for Managing Chronic Disease (SEMCD) Scale: A Scleroderma Patient-centered Intervention Network (SPIN) Cohort Study. Arthritis Care Res (Hoboken) 2015 Nov 30 (E-pub ahead of print).

8. Sandelowski M. Sample size in qualitative research. Res Nurs Health 1995;18:179-83.

9. Hsieh HF, Shannon SE. Three approaches to qualitative content analysis. Qual Health Res 2005;15:1277-88.

10. Dierckx de Casterle B, Gastmans C, Bryon E, Denier Y. QUAGOL: a guide for qualitative data analysis. Int J Nurs Stud 2012;49:360-71.

J Rheumatol 2016;43:1265-7; doi:10.3899/jrheum.160602 